area and intensity in units of the local continuum was only $0 \cdot 30$. For flares with areas as great as 300 millionths, it was reduced to 0.03 , or no systematic increase of intensity with area. Area and duration of the flare appear to be more closely associated than do area and maximum intensity. On the average, large flares continue for a longer period of time than do small flares.

Comparison of the photometric light curves for flares with the times of associated sudden ionospheric disturbances, as reported by or through the Central Radio Propagation Laboratory of the United States National Bureau of Standards, indicates that generally the S.I.D. starts during the ascending branch of the flare light curve and before maximum intensity has been attained. Flare-durations based on light curves measure the period of changing $\mathrm{H} \alpha$ intensity and tend to be considerably longer than flare-durations based on visual observations. According to the durations as shown by light curves, flares near the central part of the solar disk and their associated S.I.D.'s cover very nearly the same time interval. On the other hand, S.I.D.'s accompanying flares near the limb tend to last considerably longer than the observed $\mathrm{H} \alpha$ event.

The above results have been confirmed by direct examination of records of the $5 \mathrm{Mc}$ /sec. WWV signal as obtained by the School of Electrical Engineering at Cornell University. Furthermore, examination of the Cornell records has indicated that there are numerous, well-defined, flare-associated disturbances of the ionosphere that have not hitherto been reported. This stems in part from the fact that some of the disturbances start more gradually than the usually recognized Dellinger effects. The validity of these flare-associated 'gradual ionospheric disturbances' has been confirmed by comparison with the ionospheric records of the National Bureau of Standards by members of their ionospheric staff.

\title{
3. OPTICAL OBSERVATIONS OF SOLAR FLARES
}

\section{By M. A. Ellison}

\section{Flares on the Disk}

A flare is a catastrophic event occurring on the Sun which has immediate repercussions on the Earth.

Let me illustrate some of the properties of flares by reference to three of the greatest which have been fully recorded in recent times:

(a) I942, February 28 ; time of flash, $12^{\mathrm{h}} \mathrm{OO}^{\mathrm{m}}$; duration, $>3^{\mathrm{h}} \cdot 5$; area of emission filaments, $2200 \mathrm{~m}$.; long. E. $04^{\circ}$, lat. N. $07^{\circ}$. It was this flare which led to the discovery of solar radio noise by J. S. Hey, and it also enabled the writer to detect the asymmetry in the emission $\mathrm{H} \alpha$ line (this effect having been noted independently by $\mathrm{M}$. Waldmeier in I94I). Sudden ionospheric disturbances (an intense crochet and a radio fadeout of duration $>8^{\mathrm{h}}$ ) coincided with the time of the flash. A magnetic storm, classified as great (G), began $19^{\mathrm{h}} \cdot 4$ later. This flare also gave rise to the first recorded burst of cosmic radiation $\left(+7 \%\right.$ at Cheltenham) which reached maximum intensity $\mathrm{I}^{\mathrm{h}}$ after the maximum of the flare $(x, 2)$.

(b) I946, July 25 ; time of flash, $16^{\mathrm{h}} 27^{\mathrm{m}}$; duration, $>4^{\mathrm{h}} \cdot 3$; area of emission filaments, $2500 \mathrm{~m}$. and overall length $550,000 \mathrm{~km}$.; long. E. I $5^{\circ}$, lat. N. $22^{\circ}$. Spectra photographed at Sherborne showed the $\mathrm{H} \alpha$ emission line $\mathrm{I} 6 \AA$ wide at maximum with central intensity $300 \%$ of the continuum. The continuous spectrum of the flare (intensity $10 \%$ of the adjacent continuum at $\lambda 6000$ ) was recorded at the same time. Accompanied by intense S.I.D.'s of long duration (fadeout $>5^{\mathrm{h}} \cdot 5$ ). The burst of radio noise on $72 \mathrm{Mc}$. /s. (maximum $I 6^{\mathrm{h}} 24^{\mathrm{m}}-16^{\mathrm{h}} 27^{\mathrm{m}} \cdot 5$ ), as recorded by Lovell and Banwell, surpassed the solar black body value by a factor of $10^{8}$. The associated cosmic ray burst reached its maximum intensity ( $+20 \%$ at Cheltenham) $2^{\mathrm{h}} \cdot 5$ after the flare flash. A magnetic storm, classified as very great (V.G.), began $26^{\mathrm{h}} \cdot 4$ after the peak intensity of the flare $(3,4)$.

(c) I949, November I9; time of flash, $\mathrm{IO}^{\mathrm{h}} 3^{\mathrm{m}}$; duration, $\mathrm{I}^{\mathrm{h}} 4 \mathrm{O}^{\mathrm{m}}$; area of emission, $2120 \mathrm{~m}$.; long. W. $70^{\circ}$, lat. S. $02^{\circ}$. This flare was noted for a great blow off of chromo- 
spheric material which was followed outwards to a height of $560,000 \mathrm{~km}$. above the Sun's limb. The spectra showed a maximum $\mathrm{H} \alpha$ emission line width in excess of $20 \AA$ and a central intensity of twice the normal continuum. The short-lived continuous spectrum of the flare was again recorded. The associated S.I.D.'s began at $10^{\mathrm{h}} 30^{\mathrm{m}}$, and an intense burst of radio noise on $73 \mathrm{Mc}$./s. was recorded by J. S. Hey, beginning at $\mathrm{IO}^{\mathrm{h}} 3 \mathrm{I}^{\mathrm{m}}$. This flare was not followed by a magnetic storm after the usual interval of about I day, which is in accordance with Newton's statistical law that the probability of a storm following a flare is much less when the flare is far from the centre of the disk. A burst of cosmic radiation-the greatest ever observed-reached its peak intensity $\left(+43 \%\right.$ at Cheltenham, and $+180 \%$ at Climax) $0^{\text {h }} \cdot 5$ after the flare flash $(3,5,6)$.

As these illustrations show, great flares seen on the disk usually have a well-defined filamentary structure (order of dimension of a filament I00,000 km. long by $10,000 \mathrm{~km}$. wide) and the filaments pass right across the umbra and penumbra of the associated sunspot. The filaments are less marked in smaller flares and the latter seem to avoid the locations directly above the larger spots of a group. Flares often recur repeatedly in the same location over an active sunspot( ()).

\section{Flares in Profile at the Limb}

We must combine these disk appearances with limb profile pictures in order to obtain a true understanding of flare structure. Three cases of special interest have been studied:

(a) I942, February $2 \mathrm{I} ; \mathrm{I} 3^{\mathrm{h}} 3^{\mathrm{m}}-\mathrm{I} 5^{\mathrm{h}} \mathrm{O} 7^{\mathrm{m}}$ (Meudon $\mathrm{H} \alpha$ ). This flare occurred over the same great sunspot as that described in $\mathrm{r}(a)$, and from all the attendant circumstances both were of the same order of dimensions and intensity. The brilliant emission appeared as a fringe on the upper side of a long horizontal 'prominence' and was connected by sinuous bright filaments to the chromosphere below. The emission region rose slowly to a height of $40,000 \mathrm{~km}$. before fading away (mean upward velocity $7 \mathrm{~km}$. $/ \mathrm{sec}$.). Projected length was $150,000 \mathrm{~km}$. (8). Radio fadeout and crochet, $13^{\mathrm{h}} 3 \mathrm{O}^{\mathrm{m}}-\mathrm{I}^{\mathrm{h}} \mathrm{OO} \mathrm{O}^{\mathrm{m}}$.

(b) I939, April 29; $09^{\mathrm{h}} \mathrm{O} 2^{\mathrm{m}}$ (Coimbra K). Similar in appearance to $(a)$, projected length I00,000 km., height $16,000 \mathrm{~km}$. Radio fadeout, $07^{\mathrm{h}} 46^{\mathrm{m}}-09^{\mathrm{h}} 4^{\mathrm{m}}$. Crochet, $07^{\mathrm{h}} 4 \mathrm{I}^{\mathrm{m}}$.

(c) I947, August $23 ; 16^{\mathrm{h}} 55^{\mathrm{m}}$. A dome-shaped patch of emission detached from the chromosphere at a height of $\mathrm{I} 8,000 \mathrm{~km}$., but connected laterally by thin prominence filaments to lower chromospheric levels. $\mathrm{H} \alpha$ line width of emission $>20 \AA$. Geophysical effects suggested a flare of Class 3 or $2(9)$.

From these cases, along with much similar observational material, we may summarize the characteristics of the emission regions as follows:

(I) The emission is generated over sunspot and plage regions.

(2) The emission in great flares has a structure of narrow filaments, the extension of which is greater horizontally than in a vertical direction.

(3) While the flare may originate within the chromosphere, much of its subsequent development takes place in the coronal space.

(4) The emission is virtually stationary (sightline velocities < I0 $\mathrm{km}$. $/ \mathrm{sec}$.).

(5) Adjacent regions of the chromosphere throw out prominence jets at speeds of the order of $500 \mathrm{~km}$. $/ \mathrm{sec}$. In general this high-speed material is only recorded by instruments (e.g. spectrohelioscope) which can scan \pm Io $\AA$ wave-length. Photometric observations show that this matter is brighter in $\mathrm{H} \alpha$ than that of normal prominences; though it is less bright than the stationary flare emission: the moving matter has often been confused with the emission when it has been observed near the limb, but the two phenomena are clearly distinct when seen against the disk. 


\section{Spectral Characteristics}

Most flare spectra have been obtained against the chromosphere as background. As the flare brightens, the absorption profile of $\mathrm{H} \alpha$ becomes filled in with emission, until, during the flash phase, the line width and central intensity increase catastrophically. The flash phase usually occurs within 5-1o min. of the observed time of first brightening.

Plots of line width or of central intensity versus time, relating to the brightest part of the flare, are known as development curves. These plots still form the best available method of indicating the life-history of a flare and of its light output in $\mathrm{H} \alpha$.

The duration of the flare flash is no more than 2-3 min., even in those great flares which last for several hours, and it is during this short period that the spectrum is of supreme interest. The continuous emission spectrum is confined to this stage $(4,5)$. Likewise, the well-known geophysical effects of flares (sudden ionospheric disturbances, radio noise outburst and initial expulsion of particles) appear to be critically determined in time and intensity by this flash of radiation.

The cause of the sudden broadening of the hydrogen lines ( $\sim 20 \AA$ in $\mathrm{H} \alpha$ together with central intensity $200-300 \%$ of continuum) is still undecided: it may arise from the operation of the Stark effect(10), or simply as a result of a great increase in the number of excited $\mathrm{H}$ atoms coupled with strong self-absorption(ri). This question can only be investigated by further accurate photometric work on the Balmer line profiles(12). Simultaneous spectra $(\mathrm{H} \alpha-\mathrm{H} \epsilon)$ of high dispersion $(\sim 2 \AA / \mathrm{mm}$.) and corrected for scattered light, are needed during the flash. If these can be obtained for a flare outside the limb, a wealth of new material will be available.

The asymmetry of the emission $\mathrm{H} \alpha$ line observed during the flash phase (13) provides strong evidence for the expulsion of hydrogen atoms in all directions from the flare region: this effect is to be clearly distinguished from the ejection of surge prominences in specific directions at about the same time.

\section{Physical Conditions}

We have found that flares are not confined to the chromosphere, but can occur in detached prominence material lying at considerable heights within the corona.

Previous to the flare there is no observable change in the radiation field of the near-by photosphere; the flare region itself is stationary and there is no visible evidence of matter moving into it from higher or lower levels. Such motions would be easily detected with the spectrohelioscope. The spectrum indicates that the kinetic temperature of the emission region is comparable with that in a prominence $\left(\sim 10,000^{\circ} \mathrm{K}.\right)$.

The energy output of a great flare at maximum is of the order of $10^{30} \mathrm{ergs} / \mathrm{sec}$. The speed of onset for the fastest flares is such that this energy emission rate may be established over an area of $3 \times 10^{9}$ square kilometres $\left(=10^{9}\right.$ square miles) in a period of the order of I minute.

Our problem, then, is to account for the invisible transport of energy at this rate between the spot magnetic field and the flare region. Present discharge theories may be unacceptable from a theoretical standpoint $\left(\mathrm{x}_{4}\right)$; but the observational evidence is strongly in favour of some form of discharge as the cause of flares. We must remember that solar electromagnetics is a new subject and is still very incompletely understood.

\section{REFERENCES}

(I) H. W. Newton, The Observatory, 64, 260, 1942.

(2) J. S. Hey, Nature, 157, 47, 1946.

(3) S. E. Forbush, T. B. Stinchcomb and M. Schein, Phys. Rev. 79, 501, I950.

(4) M. A. Ellison, M.N.R.A.S. 106, 500, 1946.

(5) M. A. Ellison and M. Conway, The Observatory, 7o, 77, 1950.

(6) R. Bureau and A. Dauvillier, Ann. de Geophys. 6, 77, 1950. 
(7) H. W. Dodson and E. R. Hedeman, $A p . J$. x10, 242, 1949.

(8) H. W. Newton, Jour. B.A.A. 57, 54, 1947.

(9) M. A. Ellison, The Observatory, 67, 206, 1947.

(Io) M. A. Ellison and F. Hoyle, The Observatory, 67, I8I, 1947.

(r I) L. Goldberg, H. W. Dodson and E. A. Müller, Ap. J. 120, 83, I954.

(12) E. R. Mustel and A. B. Severny, Reports of Crim. Astrophys. Obs. 8, 19, 1952.

(r3) M. A. Ellison, Huitième Rapport de la Commission pour l'Etude des Relations entre les Phénomènes Solaires et Terrestres, p. 32 (Paris, I954, Sennac).

(14) T. G. Cowling, The Sun (Kuiper), p. $5^{87}$.

\section{DISCUSSION}

H. AlFvén: Could you give some figures for the density of matter in the point where the flare starts?

M. A. Ellison: It would be very difficult to give a density figure for these active regions. The flare certainly begins at very different heights.

M. N. SAHA: I want to know whether observations have been made in other spectral lines, say those of $\mathrm{He}$ ?

M. A. Ellison: We also observe very interesting effects in the $\mathrm{He} \mathrm{I}$ lines. The great need at present is to obtain simultaneous spectra in the Balmer series $(\mathrm{H} \alpha-\mathrm{H} \epsilon)$ of high dispersion and free from scattered light.

M. N. SAHA: Have lines in the Lyman series been observed during a flare?

M. Minnaert: No rocket observations have been made yet at such moments.

L. GOLDBERG: Is the dip in the red wing of $\mathrm{H} \alpha$ due to water-vapour absorption?

M. A. Ellison: Yes.

\section{THE FINE STRUCTURE OF FLARES}

\section{By A. B. Severny}

Our new tower telescope has permitted us to discover some new peculiar characteristics of the facula- and flare-emission. The most striking feature is a concentration of the emission in very small grains, like smallpox, with a size of some hundred $\mathrm{km}$. The continuous emission looks like a very thin thread along the whole spectrum. The intensity of this thread increases steadily towards the violet. Also, the line emission appears as thin threads cutting the absorption lines. Generally, only the wings of the emission lines are observed, and no reversal appears in the core, as if the core were undisturbed. I would like to draw your attention to the interesting fact that sometimes only one of the wings (on the blue side) is observed. I have introduced the term 'moustaches' for this emission. Sometimes these moustaches can be followed up to several $\AA$ from the centre of the $\mathrm{H} \alpha$ line and up to ro $\AA$ from the centre of the $\mathrm{H}$ and $\mathrm{K}$ lines.

It should be emphasized that this fine structure has a short lifetime and can be observed only at perfect seeing conditions and with an instrument of good resolving power. It seems to us that these one-sided moustaches must be considered as observational evidence for the presence of corpuscular streams. This opinion is also based on results found by Dr Nikonov and myself from a photo-electric investigation of faculae and flares made in the spectral lines $H \alpha, H$ and $K$. Sometimes a very narrow and weak $K_{4}$ emission has been found in faculae and flares observed at the limb. If we consider an additional depression in the blue wing mentioned above as due to a cloud of $\mathrm{Ca}+$ particles ejected from the flare, the particle density will be $10^{4} \mathrm{~cm}^{-3}$. The position of this depression corresponds to velocities of about $1000 \mathrm{~km}$. $/ \mathrm{sec}$.

According to our spectroscopic observations the continuous emission and the moustaches are pronounced in the very first stage of development of flares and faculae. The second and especially the post-maximum stage is characterized, as is well known, by a strong and broad line-emission which sometimes consists of thin threads too. 